\title{
Electron Dynamics in a DNA Molecule
}

\author{
V.M. Apalkov ${ }^{\dagger}$ and Tapash Chakraborty ${ }^{* \ddagger}$ \\ ${ }^{\dagger}$ Department of Physics and Astronomy, Georgia State University, Atlanta, Georgia 30303, USA \\ * Department of Physics and Astronomy, The University of Manitoba, Winnipeg, Canada R3T 2N2
}

(June 24, 2018)

\begin{abstract}
We report on our theoretical investigations of the electronic states in a DNA molecule. We have used a two-leg charge ladder model where electron-electron interactions and the electron spin have been taken into account. The energy spectra for G-C and A-T base pairs obtained by numerically diagonalizing the Hamiltonian reveal a gap structure and the interaction is found to enhance the energy gaps. We also present the charge distribution in the ground state and low-lying excited states for the A-T and G-C base pairs.
\end{abstract}

The unique properties of DNA, self-assembly and molecular recognition, has rendered the 'molecule of life' [1] a promising candidate in the rapidly emerging field of molecular nano-electronics [2]. A recent report of a field-effect transistor based on DNA molecules [3], that was preceded by a series of seminal experiments on the electron conduction in DNA [4], has sparked a lot of interest on the electronic properties of the DNA. A thorough understanding of the electronic properties of DNA is crucial in the development of the future DNA-based nanoscale devices. In addition, charge transfer through DNA also plays an important role in radiation damage and repair [5] and therefore important for biological processes. Following the techniques pioneered earlier for a direct measurement of electrical transport through a DNA molecule [6], Yoo et al. [3] measured the conductance of poly(dG)-poly(dC) DNA (1.7 - $2.9 \mu \mathrm{m}$ long) and poly $(\mathrm{dA})$-poly(dT) DNA $(0.5-1.5 \mu \mathrm{m})$. The bundle of DNA molecules were trapped between electrodes that were $20 \mathrm{~nm}$ apart. The I-V results showed a strong temperature dependence of the current and was interpreted in terms of a small polaron hopping model where the current was expressed as $I \propto \sinh \beta V \mathrm{e}^{-E_{a} / k T}$. Here, $E_{a}$ is the activation energy, $T$ is the background temperature, $\beta=e a / 2 k T d, e$ is the electron charge, $a$ is the hopping distance, and $d$ is the separation between the electrodes. The results for poly(dA)-poly(dT) DNA can very well be described in this way if $\beta$ is taken to be independent of temperature. In the case of poly $(\mathrm{dG})$-poly $(\mathrm{dC})$ DNA molecules, a similar temperature dependence was observed but with a much lower resistance of $1.3 \mathrm{M} \Omega$ at room temperature as compared to $100 \mathrm{M} \Omega$ for the $\operatorname{poly}(\mathrm{dA})-\operatorname{poly}(\mathrm{dT})$ DNA. The poly $(\mathrm{dG})-\operatorname{poly}(\mathrm{dC}) \mathrm{DNA}$ also shows the correct temperature dependence of $\beta$ and the temperature dependence of the current was observed down to $4.2 \mathrm{~K}$. In contrast, for the poly $(\mathrm{dA})-\operatorname{poly}(\mathrm{dT})$ DNA, temperature dependence of the current was observed down only to $50 \mathrm{~K}$.

Yoo et al. also performed I-V measurements with an additional electric field from the back of the Si substrate and measured the FET property of the back-gate type. They noted that, in the FET based on poly $(\mathrm{dA})$ poly $(\mathrm{dT})$, application of a positive gate voltage results in a larger conductance, i.e., an indication of $n$-type behavior. In the case of the DNA-FET based on poly(dG)poly $(\mathrm{dC})$, a $p$-type conducting behavior was observed.

The DNA conductivity measurements discussed above have also inspired a few theoretical studies [7-14]. These works primarily focused on the evaluation of transport through one-dimensional systems using a model Hamiltonian, or electronic structure calculations from first principles. It should be pointed out that the effect of electronelectron interactions is important for understanding the physical properties of the DNA molecules, such as excitation spectra, charge distribution and charge transport in DNA molecules. The reason for that is the following: There are a few energy scales which determine the DNA properties. The first one is the tunneling gap, or hopping integrals between nearest DNA base pairs. The typical value of these hopping integrals is $0.5 \mathrm{eV}$. The second energy scale is determined by the single-particle energy spectrum of a single DNA base pair. This is the energy between the highest occupied molecular orbital (HOMO) and the lowest unoccupied molecular orbital (LUMO), which is of the order of $1 \mathrm{eV}$ [7]. The other energy scale comes from interactions between electrons at different and at the same base pairs. This energy scale is $1 \mathrm{eV}$ for Hartree interactions and since it is close to HOMO to LUMO excitation energy the interaction can have strong effect on many-particle excitation spectra and charge distribution. At the same time the exchange interaction, which can be of the order of hopping integrals between nearest base pairs, should have strong effect on electron transport.

In this paper, we report on the electronic properties of the DNA, in particular, the influence of electron-electron interaction on the energy spectrum and the excitation gap. We model the double-stranded DNA as a two-leg charge ladder [7]. As a first approximation, we consider only the electronic degree of freedom and disregard the vibrational modes and their effects on the electronic motion [15]. The Hamiltonian of the electronic system consists of two parts: the tight-binding Hamiltonian, $\mathcal{H}_{t}$, and interaction Hamiltonian, $\mathcal{H}_{i}$. The tightbinding Hamiltonian is a two-chain Anderson Hamiltonian describing the hopping between the nearest neigh- 
bors (nearest bases) within each chain and the hopping between the two chains (within each base)

$$
\begin{aligned}
\mathcal{H}_{t}= & \sum_{i \sigma} \varepsilon_{h} a_{i, \sigma}^{\dagger} a_{i, \sigma}+\sum_{i \sigma} \varepsilon_{l} b_{i, \sigma}^{\dagger} b_{i, \sigma}+ \\
& \sum_{i \sigma} t_{h}\left[a_{i, \sigma}^{\dagger} a_{i+1, \sigma}+\text { h.c. }\right]+\sum_{i \sigma} t_{l}\left[b_{i, \sigma}^{\dagger} b_{i+1, \sigma}+\text { h.c. }\right] \\
& +\sum_{i \sigma} t_{h l}\left[a_{i, \sigma}^{\dagger} b_{i, \sigma}+\text { h.c. }\right]
\end{aligned}
$$

where $\varepsilon_{h}, \varepsilon_{l}$ are the (site) energies of the HOMO and LUMO for a single isolated base pair respectively, $a_{i, \sigma}$, $b_{i, \sigma}$ are annihilation operators of electron with spin $\sigma$ in HOMO and LUMO states of $i$-th base pair, $t_{h}$ is the hopping integral between HOMO of the nearest base pairs, $t_{l}$ is the hopping integral between LUMO of the nearest base pairs, and $t_{h l}$ is the hopping integral between strands (HOMO and LUMO) of the same base pair. The index $i$ labels the different base pairs, while $\sigma=\uparrow \downarrow$ is the spin index. The summation over index $i$ goes from 1 to $N$ where $N$ is the number of base pairs.

The interaction part of the Hamiltonian has the following form

$$
\begin{aligned}
& \mathcal{H}_{i}=\sum_{i \sigma} V_{h 0}\left(a_{i, \sigma}^{\dagger} a_{i, \sigma}\right)\left(a_{i,-\sigma}^{\dagger} a_{i,-\sigma}\right) \\
& +\sum_{i \sigma} V_{l 0}\left(b_{i, \sigma}^{\dagger} b_{i, \sigma}\right)\left(b_{i,-\sigma}^{\dagger} b_{i,-\sigma}\right) \\
& +\sum_{i \sigma \sigma_{1}} V_{h l 0}\left(a_{i, \sigma}^{\dagger} a_{i, \sigma}\right)\left(b_{i, \sigma_{1}}^{\dagger} b_{i, \sigma_{1}}\right) \\
& -\sum_{i} V_{h l 0}^{(e x)}\left(a_{i, \sigma}^{\dagger} a_{i, \sigma}\right)\left(b_{i, \sigma}^{\dagger} b_{i, \sigma}\right) \\
& +\sum_{i \sigma \sigma_{1}} V_{h 1}\left(a_{i, \sigma}^{\dagger} a_{i, \sigma}\right)\left(a_{i+1, \sigma_{1}}^{\dagger} a_{i+1, \sigma_{1}}\right) \\
& -\sum_{i \sigma} V_{h 1}^{(e x)}\left(a_{i, \sigma}^{\dagger} a_{i, \sigma}\right)\left(a_{i+1, \sigma}^{\dagger} a_{i+1, \sigma}\right) \\
& +\sum_{i \sigma \sigma_{1}} V_{l 1}\left(b_{i, \sigma}^{\dagger} b_{i, \sigma}\right)\left(b_{i+1, \sigma_{1}}^{\dagger} b_{i+1, \sigma_{1}}\right) \\
& -\sum_{i \sigma} V_{l 1}^{(e x)}\left(b_{i, \sigma}^{\dagger} b_{i, \sigma}\right)\left(b_{i+1, \sigma}^{\dagger} b_{i+1, \sigma}\right) \\
& +\sum_{i \sigma \sigma_{1}} V_{h l 0}\left(a_{i, \sigma}^{\dagger} a_{i, \sigma}\right)\left(b_{i+1, \sigma_{1}}^{\dagger} b_{i+1, \sigma_{1}}\right) \\
& -\sum_{i \sigma} V_{h l 0}^{(e x)}\left(a_{i, \sigma}^{\dagger} a_{i, \sigma}\right)\left(b_{i+1, \sigma}^{\dagger} b_{i+1, \sigma}\right),
\end{aligned}
$$

which is described by ten parameters.

In the ground state the number of electrons is equal to $2 N$, so that all HOMO states (with both spin directions) are occupied. To find the excitation gap and the energy spectrum of the electron system with the Hamiltonian Eqs. $(1,2)$, we consider the DNA structure to have a finite number $N$ of base pairs and, by exactly diagonalizing the Hamiltonian matrix, we obtain the ground state and
TABLE I. Paramaters of the DNA structure used in our present work. Energies are in $\mathrm{eV}$. The subscripts $h$ and $l$ correspond to the HOMO and LUMO.

\begin{tabular}{l|r|r}
\hline \hline $\begin{array}{l}\text { Hopping integrals } \\
{[16,17]}\end{array}$ & G-C & $t_{h}=-0.1419, t_{l}=0.0525$ \\
\hline The site energies [9] & G-T & $t_{h}=-0.0695, t_{l}=0.1054$ \\
\cline { 2 - 3 } & A-T & $\varepsilon_{h}=-14.714, \varepsilon_{l}=-14.635, \varepsilon_{l}=-13.734$ \\
\hline $\begin{array}{l}\text { On-site } \\
\text { interaction [18] }\end{array}$ & G-C & $V_{h 0}=5.879, V_{l 0}=5.227$ \\
\hline $\begin{array}{l}\text { Interstrand } \\
\text { interaction [9] }\end{array}$ & A-T & $V_{h 0}=5.681, V_{l 0}=5.23$ \\
\hline $\begin{array}{l}\text { Intrastrand } \\
\text { interaction [9] }\end{array}$ & G-C & $V_{h 1}=1.844, V_{l 1}=2.455$ \\
\hline \hline
\end{tabular}

the lowest excitation states of the system. Some of the parameters of the DNA structure relevant for our studies are listed in Table I. To eliminate the effects of boundaries we have also imposed the periodic boundary conditions, so that in Eqs. (1)-(2) we have $a_{N+1}=a_{1}$ and $b_{N+1}=b_{1}$.

We have considered a segment of DNA containing ten base pairs of poly $(\mathrm{dG})-\operatorname{poly}(\mathrm{dC})$ and poly $(\mathrm{dA})$-poly $(\mathrm{dT})$ DNA molecules. It should be pointed out that in the experiments of Porath et al. [6], the DNA sample has 30 base pairs. Similarly, we can estimate that since the distance between electrodes in the experiments by Yoo et al. [3] was about $20 \mathrm{~nm}$, taking into account the fact that the distance between the base pairs is $0.34 \mathrm{~nm}$, there were $\sim 50$ base pairs between electrodes of the set up used by Yoo et al. However, in our present model, handling more than 10 base pairs would be a formidable endeavor and has not been attempted.

From the exact diagonalization of the Hamiltonian we have obtained the energy spectra, $E_{n}$, and the corresponding wave functions, $\Psi_{n}$, of the DNA system. The energy spectra for the A-T and G-C base pairs are shown in Fig. 1. From this figure it is clear that the excitation gap for the G-C base pairs, $\Delta_{G-C} \approx 1.12 \mathrm{eV}$, is larger than for the A-T base pairs, $\Delta_{A-T} \approx 0.79 \mathrm{eV}$. In both cases the lowest excitation does not include electron spin-flip. The spin-flip excitation (open circles) gap is equal to $\Delta_{G-C \text {,spin }} \approx 1.17 \mathrm{eV}$ for the G-C base pairs and $\Delta_{A-T, \text { spin }} \approx 0.94 \mathrm{eV}$ for the A-T pairs. The main difference between the A-T and G-C pairs is that for the G-C pairs the lowest spin-flip excitations are very close to the excitations without spin reversal. For example, for the G-C pairs the energy difference between these two types of excitations is $\Delta_{G-C, \text { spin }}-\Delta_{G-C} \approx 0.05 \mathrm{eV}$, which is smaller than corresponding value for the $\mathrm{A}-\mathrm{T}$ pairs, $\Delta_{A-T, \text { spin }}-\Delta_{A-T} \approx 0.15 \mathrm{eV}$.

It is interesting to compare the energy gaps of the interacting DNA system to the corresponding gaps of the 


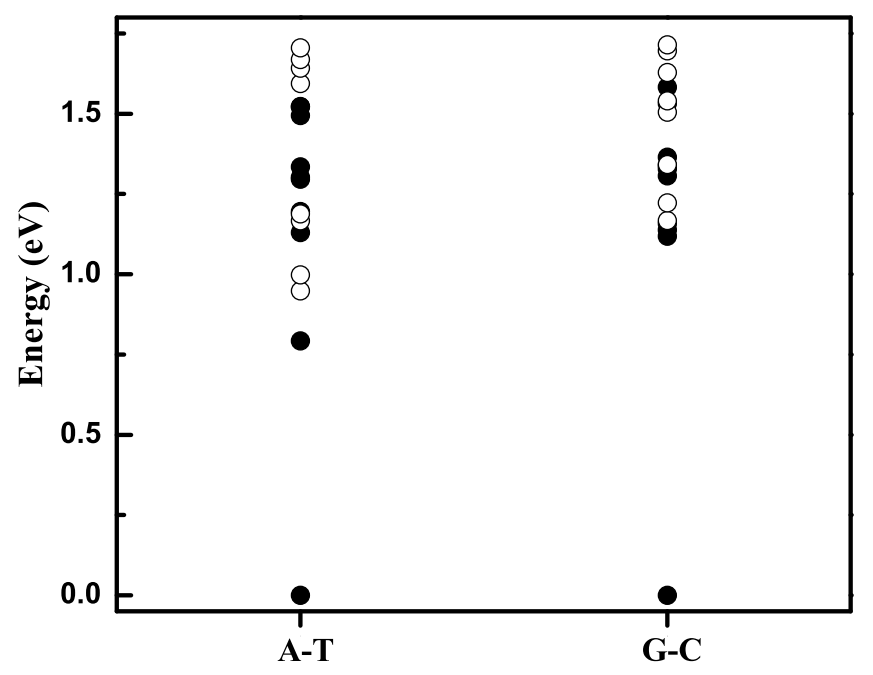

FIG. 1. The energy spectra of the A-T and the G-C base pairs in our model. The closed circles correspond to the system with equal number of up- and down-spin electrons. The open circles correspond to the spin-flip excitations.

non-interacting system. It follows from Eq. (1) that the energy gaps of the non-interacting system is equal to $\Delta_{0}=\epsilon_{l}-\epsilon_{h}-2\left(t_{l}-t_{h}\right)$, which gives $\Delta_{0, G-C} \approx 1.02$ $\mathrm{eV}$ for the G-C pairs and $\Delta_{0, A-T} \approx 0.56 \mathrm{eV}$ for the A-T pairs. Comparing these values to the energy gaps of corresponding interacting system we conclude that both for $\mathrm{G}-\mathrm{C}$ and for the A-T pairs we have interaction enhancement of the energy gaps by $0.1 \mathrm{eV}$ (for the G-C pairs) and by $0.23 \mathrm{eV}$ (for the A-T pairs). Again we see that the effect of interaction is more pronounced for the A-T pairs than for the G-C pairs. From all these results we conclude that the interaction has a weaker effect on the energy spectra for the G-C base pairs than for the A-T pairs.

In Ref. [3] the conductance of DNA molecules was found to have activated dependence on temperature. Activation energies were extracted in Ref. [3] to be $0.18 \mathrm{eV}$ for A-T base pairs and $0.12 \mathrm{eV}$ for $\mathrm{G}-\mathrm{C}$ pairs at high temperatures. These activation energies are close to our interaction enhancement of the energy gaps as described above. The activated nature of the DNA conductance means that electrons during their transport through a DNA molecule must overcome some potential barrier. Our results indicate that inter-electron interactions have considerable contribution to the activation energy of the electron transport.

The numerically generated wave functions allow us to calculate the electron charge distribution along the DNA molecule from the equations

$$
\begin{aligned}
\rho_{n, \uparrow}(k) & =\sum_{i_{1} \ldots i_{N_{\uparrow}}} \sum_{j_{1} \ldots j_{N_{\downarrow}}}\left|\Psi_{n}\left(i_{1}, \ldots, i_{N_{\uparrow}} ; j_{1}, \ldots, j_{N_{\downarrow}}\right)\right|^{2} \\
& \times \delta\left(k-i_{1}\right),
\end{aligned}
$$

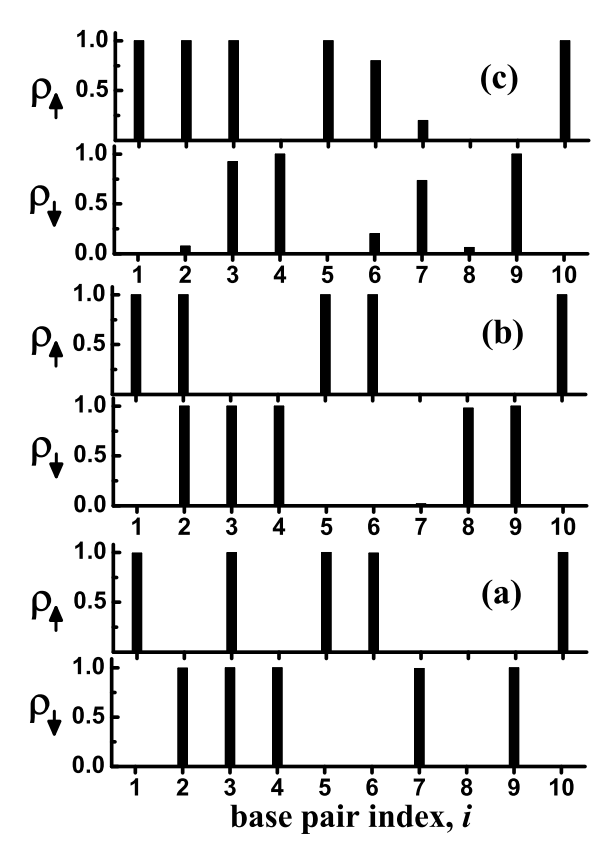

FIG. 2. The density of electrons with up- and down-spins is shown for the A-T base pairs for (a) the ground state; (b) the first excited state with equal number of up- and down-spin electrons; (c) the first spin-flip excited state.

$$
\begin{aligned}
\rho_{n, \downarrow}(k) & =\sum_{i_{1} \ldots i_{N_{\uparrow}}} \sum_{j_{1} \ldots j_{N_{\downarrow}}}\left|\Psi_{n}\left(i_{1}, \ldots, i_{N_{\uparrow}} ; j_{1}, \ldots, j_{N_{\downarrow}}\right)\right|^{2} \\
& \times \delta\left(k-j_{1}\right)
\end{aligned}
$$

where $\rho_{n, \sigma}(k)$ is the density of the electrons with spin $\sigma=\uparrow$ or $\downarrow$ in the state $n$ at the base pair $k$, and $\Psi_{n}\left(i_{1}, \ldots, i_{N_{\uparrow}} ; j_{1}, \ldots, j_{N_{\downarrow}}\right)$ is the wave function of the state $n$ with $N_{\uparrow}$ electrons with spin $\sigma=\uparrow$ and $N_{\downarrow}$ electrons with spin $\sigma=\downarrow$. Here $i_{1}, \ldots, i_{N_{\uparrow}}$ and $j_{1}, \ldots, j_{N_{\downarrow}}$ are the coordinates of electrons in the base-pair representation. The results for the charge density of the different base pairs are shown in Fig. 2 (for A-T) and in Fig. 3 (for G-C).

Clearly, for the A-T and the G-C pairs the charge distribution in the ground state is the same, which indicates that the electrons are strongly localized at the base pairs, i.e. at each base pair the electron density is equal to either 0 or 1 (the difference from 0 or 1 is less than 0.01 ). However, the charge distribution in the excited states of G-C and A-T systems show a different behavior (see Fig. 2b and Fig. 3b). For the G-C pairs, distribution of electrons with spin $\sigma=\downarrow$ (Fig. 3b) is the same as in the ground state (Fig. 3a). The excitation manifests itself only in the redistribution of the electrons with spin $\sigma=\uparrow$, making the single-electron states more delocalized. For the A-T pairs the charge distribution in the excited state is different from the ground state for both spin directions (Fig. 2b). Contrary to the case of the G-C pairs, here the single-electron states remain strongly localized even in the excited state.

The spin-flip excitations for the G-C and the A-T pairs 


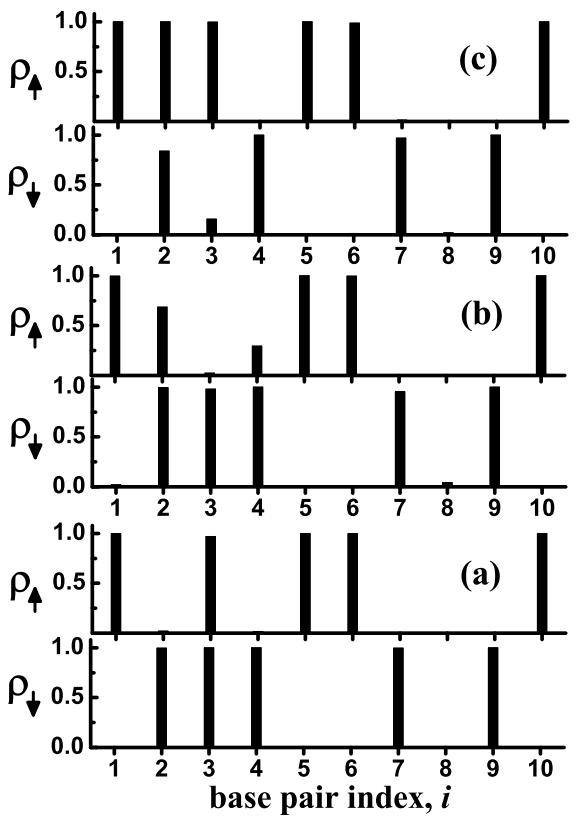

FIG. 3. Same as in Fig. 2 but for the G-C base pairs.

also behave differently. For the A-T pairs the excitation is just spin-flip at one base (with base index $i=2$ in Fig. 2c) with a weak redistribution (delocalization) of other electrons over the base pairs. For the G-C pairs the spin-flip excitation corresponds to spin-flip at one base pair with index $i=2$ (see Fig. 3c) with subsequent hopping of electron with spin $\sigma=\downarrow$ from base pair $i=3$ to the base pair $i=2$. Therefore, in the case of the G-C pairs the many-particle spin-flip excitation is a single-particle spin-flip hopping excitation. Since the electron-electron interactions tend to suppress the hopping processes, the difference in spin-flip excitations for the G-C and the A-T pairs also illustrate that the effect of electron interactions is less pronounced for the G-C base pairs than for the A$\mathrm{T}$ pairs.

In summary, we have performed theoretical calculations of the electron energy spectrum, based on a twoleg charge ladder model for the poly $(\mathrm{dA})$-poly(dT) DNA and poly $(\mathrm{dG})$-poly $(\mathrm{dC})$ DNA molecules. We take the electron-electron interactions and the electron spin degree of freedom fully into account in our model. The energy spectra for the G-C and the A-T base pairs show a large gap and the interaction was found to enhance the gap. The effect of interaction is less pronounced for the G-C base pairs than that of the A-T pairs. The spinflip excitations are not the lowest energy excitations. We also analyze the charge distribution for the ground state as well as for the excitations. The present report is the first step in our investigation of the electronic properties of the DNA. In our calculations of the energy spectra we have not included the vibrational modes. These modes are very soft in the DNA and can have strong effects on the excitation spectra, resulting in polaronic effects and strong renormalization of electron-electron interactions. Such renormalization of inter-electron interactions should in turn have strong dependence on the temperature. This is because the temperature, inducing the excitations of soft vibrational modes, strongly affect the distances between the electrons and as a result modify inter-electron interactions. The effect of vibrational modes on the energy spectra and charge distribution of DNA molecules, as well as systems containing more base pairs will be the subject of our future works.

The work of one of us (T.C.) was supported by the Canada Research Chair Program and the Canadian Foundation for Innovation Grant.

$\ddagger$ Electronic mail: tapash@physics.umanitoba.ca

[1] R.R. Sinden, DNA Structure and Function (Academic Press, 1994); C.R. Calladine, H.R. Drew, B.F. Luisi, and A.A. Travers, Understanding DNA (Elsevier, 2004), third Edition.

[2] W. Fritzsche (Ed.), DNA-Based Molecular Construction AIP Conference Proceedings, vol. 640 (2002); C. Dekker and M.A. Ratner, Phys. World 14, 29 (2001); N. Seeman, Nano Lett. 1, 22 (2001).

[3] K.-H. Yoo, D.H. Ha, J.-O. Lee, J.W. Park, J. Kim, J.J. Kim, H.-Y. Lee, T. Kawai, and H.Y. Choi, Phys. Rev. Lett. 87, 198102 (2001).

[4] For excellent review of earlier work, see e.g., H.-W. Fink, Cell. Mol. Life Sci. 58, 1 (2001); J. Richter, Physica E 16, 157 (2003); D. Porath, G. Cuniberti, and R. Di Felice, Top. Curr. Chem. 237, 183 (2004), and references therein.

[5] B. Giese, Annu. Rev. Biochem. 71, 51 (2002).

[6] D. Porath, A. Bezryadin, S. de Vries, and C. Dekker, Nature 403, 635 (2000).

[7] J. Yi, Phys. Rev. B 68, 193103 (2003).

[8] Y. Asai, J. Phys. Chem. B 107, 4647 (2003).

[9] G. Brunaud, F. Castet, A. Fritsch, and L. Ducasse, Phys. Chem. Chem. Phys. 5, 2104 (2003).

[10] G. Cuniberti, L. Craco, D. Porath, and C. Dekker, Phys. Rev. B 65, 241314 (2002).

[11] K. Iguchi, J. Phys. Soc. Jpn. 70, 593 (2001).

[12] E.I. Kats and V.V. Lebedev, JETP Lett. 75, 37 (2002).

[13] R.G. Endres, D.L. Cox, and R.R.P. Singh, Rev. Mod. Phys. 76, 195 (2004).

[14] H. Wang, J.P. Lewis, and O.F. Sankey, Phys. Rev. Lett. 93, 016401 (2004).

[15] S. Cocco and R. Monasson, J. Chem. Phys. 112, 10017 (2000).

[16] R.G. Endres, D.L. Cox, and R.R.P. Singh, preprint condmat/0201404.

[17] No data is available for hopping integrals for interchain transport, but estimated to be $t_{h l} \approx 0.01$ for GC and AT.

[18] Only Hertree terms are available [9]. 